\title{
The Management of Patients With Stage IIIA Non-Small Cell Lung Cancer With N2 Mediastinal Node Involvement
}

\author{
Renato G. Martins, MD, MPHª; Thomas A. D’Amico, MD'b; Billy W. Loo Jr, MD, PhD; \\ Mary Pinder-Schenck, MDd ; Hossein Borghaei, DO, MS ; Jamie E. Chaft, MDf; Apar Kishor P. Ganti, MD;

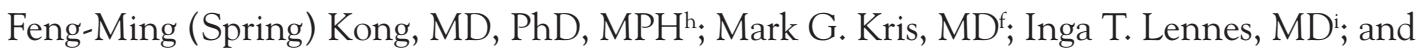 \\ Douglas E. Wood, MDª; Seattle, Washington; Durham, North Carolina; Stanford, California; Tampa, Florida; \\ Philadelphia, Pennsylvania; New York, New York; Omaha, Nebraska; Ann Arbor, Michigan; and \\ Boston, Massachusetts
}

\begin{abstract}
Patients with stage IIIA non-small cell lung cancer, determined based on involvement of ipsilateral mediastinal lymph nodes, represent the most challenging management problem in this disease. Patients with this stage disease may have very different degrees of lymph node involvement. The pathologic confirmation of this involvement is a key step in the therapeutic decision. The difference in the degree of lymph node compromise has prognostic and treatment implications. Based on multiple considerations, patients can be treated with induction chemotherapy, chemoradiotherapy followed by surgery, or definitive chemoradiotherapy without surgery. Data derived from clinical trials have provided incomplete guidance for physicians and their patients. The best therapeutic plan is achieved through the multidisciplinary cooperation of a team specialized in lung cancer. (JNCCN 2012;10:599-613)
\end{abstract}

The management of patients with stage IIIA (N2) non-small cell lung cancer (NSCLC) remains the most challenging domain in thoracic oncology, and the use of evidence-based treatment guidelines is essential. ${ }^{1}$ Notwithstanding improvements in the staging system, ${ }^{2}$ the determination of operable stage IIIA (treated with induction therapy followed by surgery) versus inoperable stage IIIA (treated with definitive chemotherapy and radiation therapy) remains controversial. Moreover, some investigators doubt the role of surgery at all, ${ }^{3}$ highlighting the importance of careful patient selection and multidisciplinary evaluation before initiation of therapy.

Another controversial issue involves the role and sequence of invasive mediastinal staging techniques, namely mediastinoscopy, endobronchial ultrasound with fine needle aspiration (EBUS-FNA), and thoracoscopy. Not only is pathologic confirmation required to confirm $\mathrm{N} 2$ disease but also adequate sampling is required to differentiate between microscopic and macroscopic (bulky) $\mathrm{N} 2$ involvement and to exclude N3 involvement.

Although the use of induction therapy has become the standard of care for potentially operable patients with stage IIIA NSCLC, the use of preoperative chemotherapy or preoperative concurrent chemoradiation therapy represents yet another potential controversial issue. ${ }^{4}$ In addition, the determination of nodal pathologic downstaging is only variably used for patient selection for surgery after induction therapy, and numerous methods exist for pathologic restaging before surgery, including repeat mediastinoscopy, EBUS-FNA, and video-assisted thoracoscopic surgery (VATS). The role of pneumonectomy after induction therapy, the choice of chemotherapeutic 
Martins et al.

agents in the induction and definitive therapy settings, the dose and technique of radiation therapy, and treatment of patients with poor performance status are other topics of debate. This article critically analyzes the most controversial issues in the multidisciplinary treatment of patients with stage IIIA (N2) NSCLC.

\section{Patients With N2 Nodal Involvement: A Heterogeneous Group}

Stage IIIA encompasses patients with a wide, heterogeneous group of tumors, ranging from small primary tumors with bulky mediastinal lymph nodes $(\mathrm{T} 1, \mathrm{~N} 2)$ to large primary tumors with adjacent tissue invasion but without lymphadenopathy (T4,N0/N1). Likewise, patients with N2 disease can have microscopic involvement, only detected at surgery, or bulky multistation involvement identified on a CT scan. Outcomes would be expected to differ significantly between these groups.

Andre et al. $^{5}$ retrospectively analyzed data on 702 consecutive patients with N2 disease at 6 French centers and found that the degree of N2 involvement correlated with prognosis. Patients with no lymph node greater than $10 \mathrm{~mm}$ on CT scan or an enlarged node but a false-negative mediastinoscopy were considered to have microscopic N2. Patients with an enlarged lymph node on CT scan were considered to have clinical N2 disease if the mediastinoscopy was positive or if it was not performed. For patients treated with primary surgery $(\mathrm{n}=562)$, the 5 -year survival rates were correlated with the degree of mediastinum involvement. $\mathrm{Pa}$ tients with microscopic positive lymph nodes in one station had the best overall survival (34\%); however, those with microscopic involvement of more than one station (11\%), clinically positive lymph nodes in one station $(8 \%)$, or multistation clinical positive nodes (3\%) all had poor outcomes. When considering these results, one must remember that these patients did not receive preoperative chemotherapy and their staging did not include a PET scan. This study also identified lymph node size as a prognostic factor, supporting the concept of bulky adenopathy in the surgical decision. Patients with positive lymph nodes measuring less than $3 \mathrm{~cm}$ had better survival (20\%) than those with lymph nodes greater than $3 \mathrm{~cm}(10 \%)$.
Investigators from the University of Alabama analyzed their single-institution experience in patients staged with PET scan. Patients with a negative $\mathrm{CT}$ and PET scan, RO resection, complete thoracic lymphadenectomy, and pathologic N2 were included. Among the 148 patients evaluated, 93\% received adjuvant chemotherapy and $13 \%$ adjuvant radiotherapy. The 5 -year survival rate of this group was $35 \%$. This study is one of the bases for the NCCN recommendation that "patients with occult positive N2 nodes discovered at the time of pulmonary resection should continue with the planned resection along with formal mediastinal LN dissection." Evidently, this recommendation assumes that the preoperative staging described in the guidelines is followed.

\section{Initial Evaluation}

Initial evaluation of patients with locally advanced NSCLC requires an assessment of the patient's cardiac and pulmonary status in addition to the staging studies. The NCCN Clinical Practice Guidelines in Oncology (NCCN Guidelines) for NSCLC recommend pulmonary function tests (PFTs) as part of the initial evaluation of patients with stage III NSCLC (to view the most recent version of these guidelines, visit the NCCN Web site at NCCN.org). This will be necessary whether surgery or radiotherapy is the preferred local therapy. Imaging studies to determine clinical staging are also indicated, including CT of the chest, liver, and adrenal glands; a PET or PET/ CT scan; and a brain MRI. Brain MRI is necessary even when the patient has no neurologic symptoms, because the rate of asymptomatic metastatic disease to the brain in stage III is approximately $20 \%$.

The management of patients with stage IIIA NSCLC requires a precise evaluation of the mediastinum, and only with that information can the optimal therapy can be determined. The initial clinical evaluation using radiographic data leads to a presumptive clinical stage in most patients. Noninvasive imaging studies can provide evidence of mediastinal lymph node involvement; however, pathologic confirmation is necessary as a rule. One of the most important avoidable errors in lung cancer staging is clinical overstaging of the mediastinum without pathologic confirmation. Patients commonly have false-positive lymph nodes from a local inflammatory response, with or without obstructive pneumonia. In rare cases, instead 
of tumor involvement, a sarcoid-like lymph node reaction with significant adenopathy and marked fluorodeoxyglucose uptake on PET imaging can be found. Many minimally invasive techniques are now available for confirming mediastinal lymph node status (e.g., EBUS, endoscopic ultrasound [EUS], mediastinoscopy, VATS); the confirmation of positive N2 lymph nodes should be routine in most cases, unless incontrovertible evidence exists of nodal or other advanced disease. However, negative N3 lymph nodes are also important to document when planning multimodality therapy. The presence of N2 disease is a predictor of the possible existence of occult N3 disease. N3 involvement would indicate stage IIIB, surgery would be excluded, and chemoradiotherapy (CRT) would be the appropriate therapy, assuming the absence of distant disease. Therefore, pathologic staging of the mediastinum must include thorough sampling of $\mathrm{N} 3$ nodes, regardless of which biopsy technique was used.

In a prospective study that evaluated the diagnostic values of PET scan and EBUS-guided transbronchial needle aspiration (EBUS-TBNA) in 117 patients with potentially operable NSCLC, the sensitivity and specificity of PET scan was 70\% and 59.8\% versus $90 \%$ and $100 \%$ for EBUS. The accuracy of PET scan was $62.4 \%$ versus $97.4 \%$ for EBUS. The negative predictive value was also higher for EBUS then for PET scan. ${ }^{8}$

An analysis of a trial comparing PET/CT with conventional imaging in the diagnostic workup of patients with early-stage NSCLC compared the accuracy of PET and invasive techniques for mediastinal staging. ${ }^{9}$ The imaging study had a sensitivity of $70 \%$ and specificity of $94 \%$. Among the 22 patients with PET interpreted as positive for mediastinal nodes, 8 did not have nodal involvement. This study confirmed that mediastinum assessment with radiographic studies alone is associated with a clinically relevant false-positive rate, highlighting the need for pathologic confirmation.

\section{Importance of a Multidisciplinary Evaluation}

A common mantra in cancer treatment is the desire for "multidisciplinary evaluation and management," but no place is this more important than in the treatment decisions for patients with stage IIIA lung can- cer. Although the politically correct expression of "multidisciplinary care" is routinely recommend, a medical oncologist is in fact rarely needed in the treatment of stage IA NSCLC and, conversely, a thoracic surgeon has little to offer patients with stage IV lung cancer. As evidenced by the presence of this article and a complex set of NCCN recommendations, stage IIIA lung cancer is complicated and heterogeneous, with variable evidence-based data and nuanced decision-making. It is the very reason these authors attempt to summarize the data and make meaningful recommendations to the many thoracic surgical, medical, and radiation oncologists trying to define rational treatment strategies for the frequent patient with locally advanced lung cancer.

The most common treatment decisions for patients with N2 NSCLC are induction chemotherapy, CRT followed by surgical resection, or definitive CRT without surgery. The decision-making between these options is carefully reviewed in this paper, but the one constant in all treatment decisions is the need for systemic therapy, and therefore the consistent need for medical oncology involvement throughout the treatment planning. Radiation oncologists may be excluded from the initial evaluation if the intent is induction chemotherapy followed by surgery, and thoracic surgeons may be excluded when definitive CRT is planned. However, in most cases a surgeon is an important part of this decision for the patient and the multidisciplinary team. Thoracic surgeons are also not commonly involved in the initial evaluation of patients being considered for induction therapy followed by surgery, with the rationale that surgeons are not needed until surgical planning is required after completion of induction therapy. However, the fallacy of this approach is that the preinduction evaluation is an important part of the surgical decision-making, the surgeon may make important recommendations regarding the treatment plan or may deem the patient inoperable from the start, completely redirecting the therapeutic plan.

Thoracic surgeons bring many important elements to the evaluation of patients with clinical stage IIIA lung cancer. First, they provide an important opinion regarding the validity of the apparent clinical staging, and the need and technique for verifying mediastinal lymph node involvement and excluding more-advanced (stage IIIB) disease that would preclude surgery altogether. Second, they are important 
Martins et al.

decision-makers regarding the role of surgery for selected patients with N2 disease. The thoracic surgeon may be an important advocate for the consideration of surgery in patients with limited N2 disease, or may influence decisions away from surgery in patients with more extensive nodal disease or with comorbidity that substantially impacts the risks and benefits of surgery. Third, they may help direct the extent of induction therapy, or the extent of pretreatment or postinduction staging, to better evaluate the appropriateness and risks of surgery. Finally, surgical discussions and consideration should be made early to best prepare the patient for the complex sequence of planned therapy. The important principle is that these decisions must be made at the outset of treatment planning, before initiation of therapy, to appropriately evaluate and educate the patient, plan any additional workup, and provide clear and mature decision-making for the therapeutic plan.

Substantial evidence now shows the improved short- and long-term outcomes of specialty surgical care for patients with lung cancer. Board certified thoracic surgeons performing lung cancer surgeries have been shown to provide decreased operative morbidity and mortality, decreased hospital length of stay and cost, and improved cancer survival compared with general surgeons. ${ }^{10}$ Although no studies have further stratified these benefits by stage, these differences are likely magnified in the subset of patients with stage IIIA lung cancer. First, the decision-making is complex in these patients and the surgeon must have a thorough and updated knowledge of the oncology literature, and a mature and trusted relationship with the medical and radiation oncology team to provide the best input on the consideration of surgery. Second, patients with locally advanced tumors demand more technical experience and precision. These patients usually have larger and more central tumors that less-experienced surgeons may incorrectly consider inoperable or require a pneumonectomy. Furthermore, these patients also have mediastinal nodal disease that requires thorough lymph node staging and resection that is most reliably accomplished by surgeons who have thoracic oncology as a dominant part of their surgical practice.

Finally, in many tertiary referral centers, the discussion among surgical, medical, and radiation oncologists occurs in the context of the multidisciplinary clinic, where the patient has the oppor- tunity to see all 3 specialists in the same visit, and at a thoracic tumor board meeting, where pathology and radiology are also reviewed. These direct interactions are very important to achieve the best possible outcome.

\section{Results of Phase II Trials Exploring Multimodality Therapy}

The poor survival of patients with stage IIIA NSCLC treated with surgery only, even when resections are complete, have led to decades of investigation to improve on these outcomes. Preoperative approaches that have been studied include chemotherapy alone, concurrent CRT, and sequential chemotherapy and radiation therapy. Trials of these preoperative modalities have yielded promising results, with apparent improvements in overall survival but increased morbidity and mortality, especially with trimodality therapy when a pneumonectomy is required. ${ }^{3}$

Much can be learned from the more than 1300 patients studied. Tables 1 and 2 summarize phase II data evaluating preoperative therapy in patients with clinical stage III NSCLC. Trials included are nonrandomized, evaluating 30 patients or more. Because some trials include stage IIIB patients, the number of patients with N2 NSCLC per trial is listed. Only one of these trials did not use cisplatin, and none used carboplatin. In fact, all the phase III data described in the next section of this paper are also based on cisplatin. Consequently, for patients who cannot tolerate the toxicities of cisplatin, no data are available to adequately guide therapy.

A total of 755 patients were treated in 11 trials assessing neoadjuvant chemotherapy, and 627 patients were treated in 11 trials assessing concurrent CRT. The overall radiographic response rates for both modalities are impressive, ranging from $64 \%$ to $81 \%$ for chemotherapy and $48 \%$ to $84 \%$ for CRT. The percentage of patients proceeding to surgical exploration was variable, with a median of 79\% (range 48\%-90\%) in the chemotherapy-only trials and a median of $61 \%$ (range 30\%-90\%) in the CRT trials. The median overall survival of 19 months for all patients in the chemotherapy-only trials was numerically superior to the 16 months seen in the CRT trials, whereas the treatment-related morbidity was higher with the addition of radiotherapy. A median of $5 \%$ treatment-related deaths was reported in the chemotherapy trials 
Management of Stage IIIA (N2) NSCLC

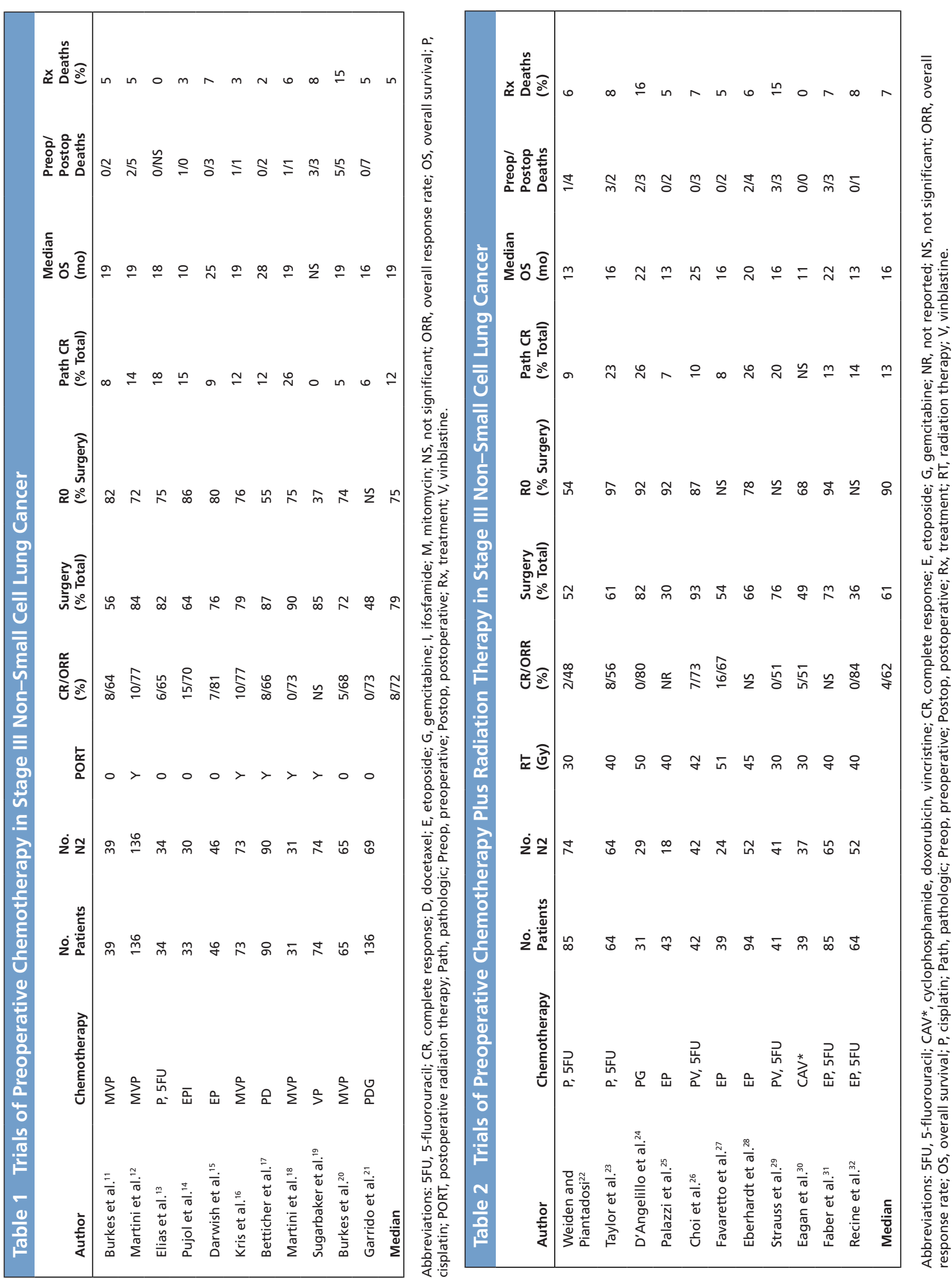


Martins et al.

and $7 \%$ in the CRT trials. In summary, the addition of chemotherapy or CRT to standard surgical resection in stage III NSCLC leads to superior survival compared with historical results. Each additional modality increases toxicity and risk of treatment-related mortality. No trend is seen toward better outcomes with the addition of induction radiation to chemotherapy. However, a trend is seen toward more perioperative and treatment-related deaths when radiation was added.

\section{Results of Randomized Phase III Trials in Stage IIIA NSCLC and Caveats on Their Interpretation}

To improve on the overall poor results for stage IIIA N2 NSCLC when treated with surgery alone, different therapeutic modalities have been evaluated in randomized clinical trials. The crucial questions that randomized trials in stage IIIA N2 NSCLC have sought to address include determining the optimal induction strategy for patients considered for surgery (chemotherapy vs. chemoradiation); defining the role for surgical resection after induction therapy; and identifying appropriate criteria for selecting patients for surgery versus definitive chemoradiation. The following section discusses results of large randomized trials in stage IIIA N2 NSCLC. Trials in stage IIIA N2 NSCLC have had challenges, including heterogeneous patient populations, imprecise staging, and slow patient accrual, which have made the results difficult to interpret and translate into uniform clinical practice.

\section{Optimal Induction Strategy}

Induction chemotherapy has been compared with CRT in stage III NSCLC. ${ }^{33}$ Between 1995 and 2003, the German Lung Cancer Cooperative Group (GLCCG) randomized 558 patients with stage IIIA and IIIB NSCLC to either preoperative chemotherapy followed by surgery followed by radiation (control arm) or preoperative chemotherapy followed by concurrent carboplatin, vindesine, and twice-daily radiation followed by surgery (interventional arm; Figure 1). Patients in both groups were predominantly men with a median age of 59 years and most had an ECOG performance status of 0 . Both the control and interventional arms included mostly patients with stage IIIB NSCLC (64\% and 69\%, respectively), although a large proportion of those classified as IIIB at that time $(43 \%$ in the interventional arm and $49 \%$ in the control arm) had $\mathrm{T} 4, \mathrm{NO}-1$ tumors, which have been reclassified as stage IIIA under the current lung cancer staging system. ${ }^{2}$

In both groups, patients were assessed for response before surgery and only those without tumor progression were considered for resection. Higher rates of resectability, mediastinal downstaging, and histopathologic tumor regression were observed in patients treated with induction CRT compared with induction chemotherapy. However, no significant differences in progression-free or overall survival were observed between the treatment strategies, either in the intention-to-treat population (Table 3) or the patients who actually underwent surgical resection (Table 4). Among patients who underwent complete resection, rates of mediastinal downstaging (N2-N3 to N0-N1) were higher in the interventional group (46\%) compared with the control group (29\%; $P=$ $.02)$. Similarly, tumor regression greater than $90 \%$ was observed in $60 \%$ of patients in the interventional group compared with $20 \%$ in the control group $(P<$ $.0001)$. A trend toward increased surgical mortality was observed in patients in the interventional group ( $9 \%$ vs. $5 \%$ in the control group; $P=.11$ ). Pneumonectomy was performed in $35 \%$ of those who underwent resection in both treatment groups; mortality after pneumonectomy was $14 \%$ in the interventional group versus $6 \%$ in the control group, a difference that was not statistically significant.

The GLCCG study has been criticized on several issues related to the study design. Many patients included in the study and deemed potentially resectable would be considered unresectable according to current practice, including those with $\mathrm{T} 4$ tumors and mediastinal nodal involvement or any $\mathrm{T}$ stage and $\mathrm{N} 3$ involvement. These patients constituted a substantial proportion of participants in this study, and their inclusion likely accounts for the large number of patients $(44 \%)$ who never went on to resection. Although the study was designed to answer the question of whether induction chemoradiation is superior to induction chemotherapy followed by postoperative radiotherapy, significant differences in the chemotherapy and radiation treatment regimens between the groups may have muddied this question. In particular, the use of twice-daily (hyperfractionated) radiotherapy in the interventional group is contro- 
versial, given that hyperfractionated radiotherapy is not standard in NSCLC and introduces an additional independent variable. The control group received conventionally fractionated radiation postoperatively. Patients in the investigational arm also received more chemotherapy than those in the control group, both in terms of cycles and agents. Finally, the use of outdated radiotherapy techniques in this trial limits the applicability of the results.

The optimal induction regimen for patients with potentially resectable stage IIIA N2 NSCLC remains an open question. Given the negative progressionfree and overall survival results despite higher rates of mediastinal downstaging and pathologic response, both chemotherapy and chemoradiation are considered reasonable induction strategies, albeit for a much more selected population of patients with stage IIIA N2 NSCLC than in the GLCCG study, and both strategies continue to be evaluated in clinical trials. As in the GLCCG study and other trials in stage IIIA NSCLC, timely accrual of patients continues to be a challenge. The Radiation Therapy Oncology Group
(RTOG) 0412/SWOG 0332, also designed to compare induction chemotherapy with induction chemoradiation before surgery in patients with $\mathrm{N} 2$ disease, was closed early because of poor accrual. Two trials comparing induction chemotherapy with induction chemoradiation are ongoing. ${ }^{34,35}$

\section{Role of Surgery}

Two large randomized controlled clinical trials have investigated the role of surgery after induction therapy in patients with NSCLC and N2 nodal involvement. ${ }^{3,36}$ The EORTC compared surgery with radiotherapy in patients who had experienced response to 3 cycles of induction platinum-based chemotherapy (Figure 2). The overall response rate to chemotherapy was $61 \%$. Among patients assigned to radiotherapy, 93\% received radiation, although compliance to radiotherapy as prescribed by the protocol was only $55 \%$. Of patients assigned to surgery, 92\% underwent the procedure. A complete resection was achieved in 50\% of patients who underwent surgery; exploratory thoracotomy was performed in $14 \%$. Pathologic complete response was observed in $5 \%$ of patients, and medias-

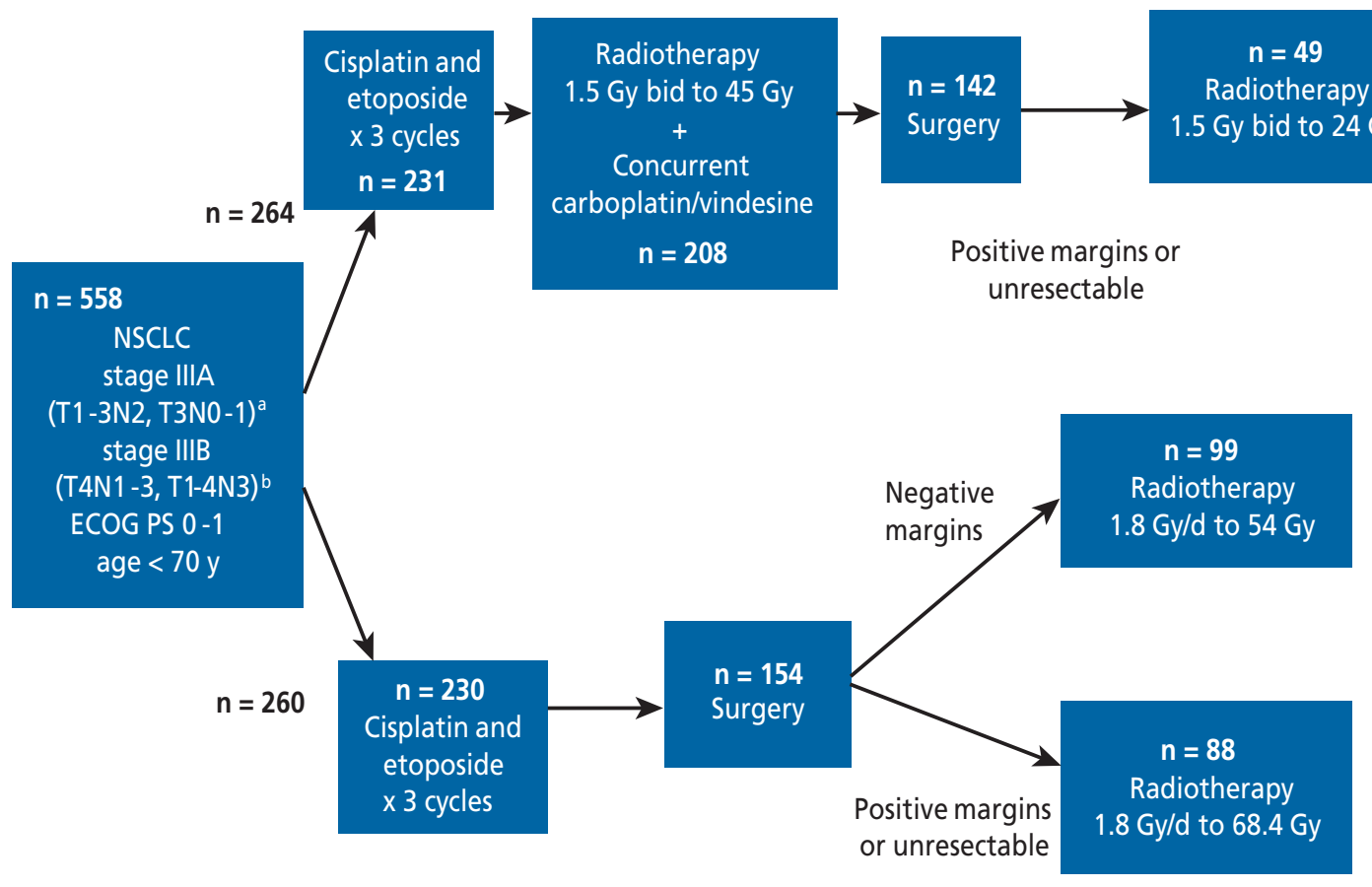

Figure 1 German Lung Cancer Cooperative Group (GLCCG) trial design.

Abbreviations: NSCLC, non-small cell lung cancer; PS, performance status.

anitial staging included CT of the thorax, abdomen, and brain; bone scan; and mandatory assessment of mediastinal lymph nodes using mediastinoscopy, thoracotomy, thoracoscopy, or needle biopsy.

bPatients with T4 tumors were eligible if tumor involved the superior vena cava, left atrium, carina, distant trachea, or the great vessels. Patients with invasion of the heart, esophagus, vertebra, malignant effusion, or supraclavicular lymph node involvement were ineligible. 
Martins et al.

\section{Table 3 Clinical Outcomes in Patients Eligible for Treatment}

\begin{tabular}{|llll|}
\hline Clinical Outcome & Interventional Group (95\% Cl) & Control Group (95\% Cl) \\
\hline Median progression-free survival (mo) & $9.5(8.3-11.2)$ & $10.0(8.9-11.5)$ & $P$ \\
Median overall survival (mo) & $15.7(13.4-18.0)$ & $17.6(14.4-20.3)$ & .87 \\
5-year survival & $21 \%$ & $18 \%$ & .97 \\
\hline
\end{tabular}

tinal downstaging occurred in $42 \%$. Pneumonectomy was performed in $47 \%$ of patients who underwent surgery. Overall operative mortality was $4 \%$; no deaths occurred in patients who underwent lobectomy, compared with a $7 \%$ mortality rate for pneumonectomy. Postoperative radiotherapy was administered to $40 \%$ of patients in the surgical arm.

Median overall survival was 16.4 months for patients who underwent surgical resection, compared with 17.5 months in those who underwent radiotherapy (hazard ratio [HR], 1.06; 95\% CI, 0.84-1.35; $P=.6$ ). Median progression-free survival was 9 months in the surgery arm and 11.3 months in the radiotherapy arm (HR, 1.06; 95\% CI, 0.85-1.33; P = .6). Locoregional relapse was more common in patients who underwent radiotherapy, whereas distant metastases were more frequent in those who underwent surgery. In an unplanned subgroup analysis of patients who underwent surgery, better survival was observed if nodal downstaging occurred, complete resection was achieved, or lobectomy was performed. ${ }^{36}$

Based on the absence of a survival benefit with surgery and lower treatment-related mortality with radiotherapy, the EORTC 8941 investigators concluded that radiation is the preferred locoregional treatment for patients with stage IIIA N2 NSCLC. However, several factors make it difficult to apply the results of EORTC 8941 to current clinical practice. Although pathologic confirmation of $\mathrm{N} 2$ involvement was required for inclusion, clinical staging of patients in this study would be considered inadequate by current stan- dards. PET/CT and brain imaging were not performed in the initial staging. PET/CT has been shown to detect occult metastases in up to $25 \%$ of patients with clinical stage III disease. ${ }^{37,38}$ Similarly, as previously stated, the incidence of asymptomatic brain metastases is high in patients with NSCLC.

Given the limited staging performed, EORTC 8941 likely included patients who would have been upstaged by current imaging techniques. Patients in this category clearly would not have been appropriate for definitive local therapy treatment, and their inclusion dilutes any possible benefit of these therapies. Similar to other trials in stage IIIA NSCLC, EORTC 8941 accrued slowly, and, in fact, was closed prematurely because of slow accrual. Patients were accrued over 8 years at 41 different institutions, with most institutions contributing small numbers of patients and a large number of surgeries performed by nonthoracic surgeons. The trial has been criticized for the high rate of pneumonectomy and low rate of complete resection. ${ }^{3}$ The high pneumonectomy rate in EORTC 8941 may have reflected the prevailing surgical practice at the time and the advanced nature of the tumors included in the study. Nevertheless, the extent of surgery remained a powerful prognostic factor in the trial. Finally, patients in the control arm of the study were treated with sequential chemotherapy followed by radiation, which has been shown to be inferior to concurrent therapy. ${ }^{39,40}$

In the North American Intergroup study (INT01393; Figure 3), patients with stage IIIA N2

Table 4 Clinical Outcomes in Patients Undergoing Resection

\begin{tabular}{|c|c|c|c|}
\hline Clinical Outcome & $\begin{array}{l}\text { Interventional Group (95\% Cl) } \\
\mathrm{n}=131\end{array}$ & $\begin{array}{l}\text { Control Group }(95 \% \mathrm{Cl}) \\
\mathrm{n}=141\end{array}$ & $P$ \\
\hline $\begin{array}{l}\text { Median progression-free } \\
\text { survival (mo) }\end{array}$ & $19.6(14.8-27.1)$ & $21.3(14.7-29.9)$ & .64 \\
\hline Median overall survival (mo) & $32.4(21.3-50.0)$ & $33.0(25.7-44.8)$ & .54 \\
\hline 5-year survival & $39 \%$ & $31 \%$ & \\
\hline Complete resection & $75 \%$ & $60 \%$ & .008 \\
\hline
\end{tabular}




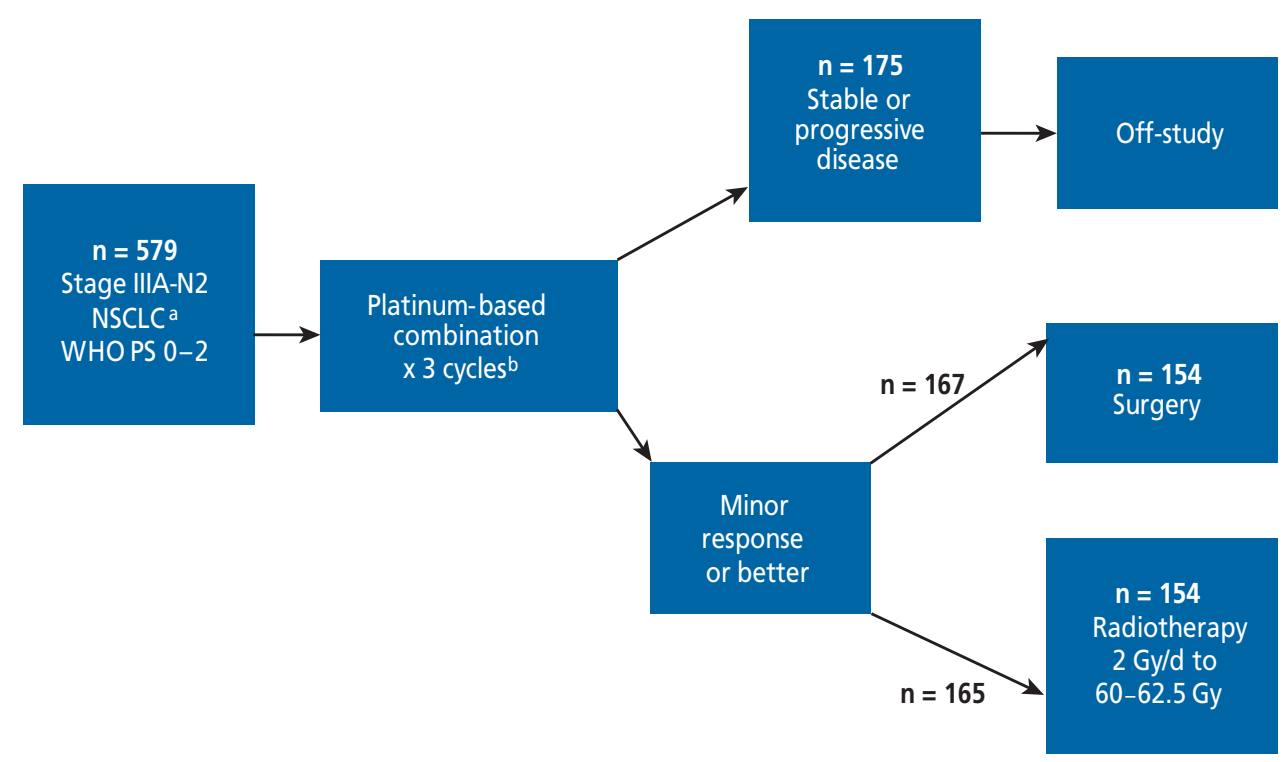

Figure 2 EORTC 8941 trial design.

Abbreviations: NSCLC, non-small cell lung cancer; PS, performance status.

anitial staging included CT of the thorax, CT or ultrasound of the abdomen, and cytologic or histologic proof of N2 involvement. For nonsquamous cancers, patients with any N2 involvement were eligible. For squamous cancers, N2 nodal involvement exceeding level $4 \mathrm{R}$ for right-sided tumors and levels $5 / 6$ for left-sided tumors was required.

${ }^{\mathrm{b}}$ Chemotherapy consisted of either cisplatin $>80 \mathrm{mg} / \mathrm{m}^{2}$ or carboplatin at an area under the curve of at least 5 combined with at least one other drug. Most patients received either platinum/gemcitabine or platinum/taxane combinations.

NSCLC were randomized to either concurrent induction chemotherapy (2 cycles of cisplatin and etoposide) plus radiotherapy (45 Gy) followed by resection or to the same induction therapy followed by completion of radiotherapy uninterrupted up to $61 \mathrm{~Gy}$. Patients were assessed for response with a CT scan after induction therapy, and those without tumor progression then either underwent surgery or definitive radiotherapy. The median age was 60 years and most patients had an excellent performance status, less than $5 \%$ weight loss, and involvement of only one nodal station.

Median overall survival did not differ significantly between the groups, at 23.6 months for the surgical group versus 22.2 months for the radiotherapy group (HR, 0.87; 95\% CI, 0.70-1.10; P = .24). The surgical group had superior progression-free survival at 12.8 months, compared with 10.5 months in the radiotherapy group ( $\mathrm{HR}, 0.77 ; 95 \% \mathrm{CI}, 0.62-0.96$; $P=.017$ ). Grade 3 or 4 esophagitis was more common in the group treated with higher doses of radiation. Grade 3 or 4 pneumonitis was also significantly more common in the nonsurgical group. Treatment-related deaths were higher in the surgical group $(8 \%)$ than in the radiotherapy group (2\%). Most deaths in the surgical group occurred in patients who underwent pneumonectomy; 14 of 54 (26\%) of those who underwent pneumonectomy died in the perioperative period. Fewer patients in the surgical group were able to complete consolidation chemotherapy ( $55 \%$ vs. $74 \%$ in the nonsurgical group). Patients who underwent surgery were less likely to experience local-only relapse than those who underwent chemoradiation alone. In an exploratory analysis, the authors found that patients who underwent lobectomy seemed to have a superior prognosis compared with clinically matched patients treated with radiotherapy.

Strengths of INT0139 include more-comprehensive staging performed compared with previous trials; consistency of the chemotherapy regimen used within and between groups; and the administration of radiotherapy without interruption in the nonsurgical group. The study did not meet its primary end point of improved overall survival with surgery, even in a relatively select group of patients. However, the high surgical mortality in patients who underwent pneumonectomy may have offset any benefit from surgery. This study highlighted the limitations of restaging with CT scan for assessment of pathologic response: $45 \%$ of $\mathrm{pTO}, \mathrm{NO}$ specimens were resected with a pneu- 


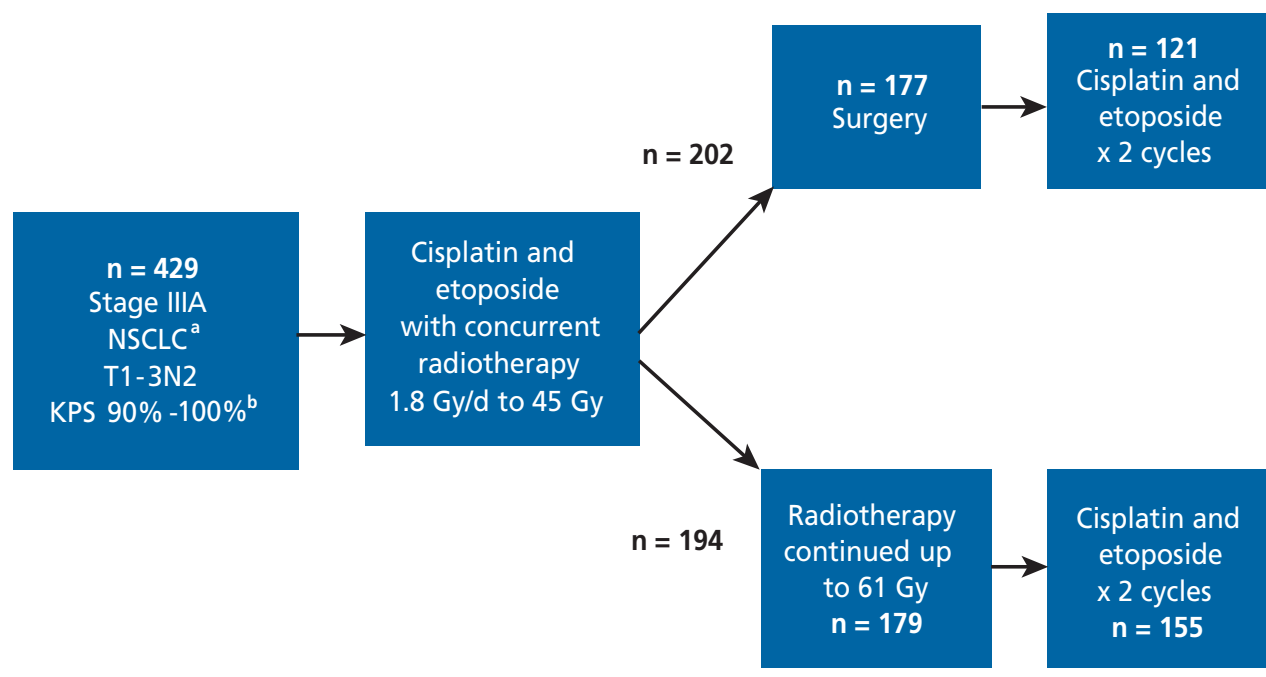

Figure 3 North American Intergroup Study (INT0139) trial design.

Abbreviations: KPS, Karnofsky perfomance status; NSCLC, non-small cell lung cancer.

${ }^{a}$ Initial staging included CT of the thorax and abdomen; bone scan; and brain MRl or CT. Pathologic proof of N2 involvement was required. Contralateral nodes $>$ then $1 \mathrm{~cm}$ were biopsied to exclude N3 disease. Patients had to be considered potentially resectable by a thoracic surgeon.

${ }^{b}$ KPS of $90 \%-100 \%$ or KPS of $70 \%-80 \%$ if albumin normal and $<10 \%$ weight loss.

monectomy. The dose of radiotherapy administered in the trial was relatively low and may have resulted in the higher rates of local failure observed in the nonsurgical arm.

To summarize, even the most robust randomized trials performed in stage IIIA N2 NSCLC have suffered from imprecise clinical staging, patient heterogeneity, slow accrual, and within-trial variation in surgical and radiotherapy methods. Subgroups with a better prognosis can be defined postsurgery (those with nodal downstaging and those who are able to undergo lobectomy). Unfortunately, a set of preoperative characteristics that allow selection of patients most likely to benefit from surgery cannot be defined currently. Furthermore, standard radiographic techniques, such as CT and PET/CT, perform poorly at predicting mediastinal lymph node clearance after preoperative therapy. ${ }^{41}$ Newer modalities, such as EBUS and EUS, ${ }^{42,43}$ may allow more accurate restaging of the mediastinum postinduction, and therefore better selection of patients who may benefit from surgery. Establishing definitively the benefit of surgery in patients with mediastinal downstaging requires a randomized trial comparing radiotherapy with surgery in patients with documented downstaging of the mediastinum after induction therapy. However, given the historically poor accrual to randomized trials in stage III NSCLC and the currently limited availability of techniques such as EBUS and EUS, a study such as this might prove infeasible.

\section{Radiation Therapy Technique Recommendations}

Rapid advances in radiation therapy technology have enabled conformal dose sculpting, dose intensification, and normal tissue sparing beyond what had been possible when much of the knowledge from clinical trials of combined modality therapy was acquired. Therefore, important questions remain on the role and optimization of radiation therapy in multimodality therapy for resectable stage IIIA NSCLC. The question of the role of radiation therapy in preoperative therapy was discussed previously. This section addresses the issues of the appropriate dose and target volume in this setting. Neither of these important questions has been answered directly in comparative clinical trials. Consequently, current recommendations based on available evidence and consensus clinical judgment are summarized.

The major randomized trials using preoperative $\mathrm{CRT}^{3,33}$ and multiple phase II trials used radiation therapy doses of 45 to $50 \mathrm{~Gy}$, historically considered to be appropriate for sterilizing microscopic disease burden, with postoperative boost doses reserved for positive margins or gross residual disease. Consistent with this, radiation therapy in most of these studies 
targeted at least some elective mediastinal nodal volumes at risk of harboring micrometastases. When administered postoperatively, radiation therapy doses after complete resection tend to be slightly higher at 50 to 54 Gy in most series. ${ }^{44}$ For either pre- or postoperative radiation therapy, nodal targets can be extrapolated from known patterns of nodal spread ${ }^{45-47}$ and regional failure after surgery for earlier-stage NSCLC..$^{48}$ Higher doses have been avoided historically because of the higher risk of postoperative complications, such as bronchopleural fistula and respiratory distress. ${ }^{49}$

This raises an important point in the management of these patients: patients treated with preoperative CRT will usually receive radiation doses inferior to those they would have received with definitive CRT. If a determination of resectability is to be made after a trial of preoperative CRT, this decision must be made through timely restaging and by a thoracic surgeon working in the context of a multidisciplinary team to permit completion of definitive CRT with no or minimal treatment break if the tumor is deemed unresectable at that point. If this team is not in place and a treatment break of no more than a few days is not possible, a trimodality strategy should not be used unless resectability can be assessed confidently before any therapy.

To address this issue, higher definitive doses of preoperative CRT have been administered safely when using specific operative techniques to minimize the potential complications, including reinforcing the bronchial stump with a muscle flap and limiting intraoperative and postoperative fluid administration and mechanical ventilation barotrauma. ${ }^{50,51}$ The RTOG completed a multi-institutional phase II study of preoperative high-dose (61.2 Gy) CRT with mandated surgical quality assurance (RTOG 0229), finding a promising $63 \%$ rate of mediastinal pathologic complete response and median overall survival of 26.6 months without excessive toxicities..$^{52}$ Potential advantages of this approach include the possibility of even higher mediastinal clearance rates and a reduced risk of interrupting definitive CRT if surgery is ultimately infeasible, but whether it will result in superior survival over more conventional pre- or postoperative doses remains to be tested. Targeting only involved nodal levels is reasonable to minimize toxicity in the context of treatment intensification, analogous to the current predominant practice of involved-field irradiation in definitive CRT.

\section{Recommendations}

The appropriateness of surgical resection in stage IIIA NSCLC ideally should be determined before initiation of all therapy, because preoperative therapy does not reliably increase the resectability of initially unresectable disease. Preoperative radiation therapy doses of 45 to $50 \mathrm{~Gy}$ and postoperative doses of 50 to 54 Gy are standard with complete resection. Customized targeting of the highest-risk nodal regions based on tumor location, such as recommended in the Lung Adjuvant Radiotherapy Trial (Lung ART) protocol, ,3 $^{3}$ is reasonable, although practice varies considerably. An experienced multidisciplinary thoracic oncology team is paramount in the setting of multimodality therapy for stage IIIA NSCLC. This is particularly true if definitive radiation therapy doses are to be administered in combination with surgery, and target volumes should be more limited in this setting. Modern 3-dimensional conformal radiation therapy techniques should be used to minimize unnecessary normal tissue irradiation, and more-advanced technologies to conform dose or manage respiratory motion are appropriate when required to meet normal tissue dose constraints if proper quality assurance measures are implemented (see NCCN Guidelines for NSCLC, ${ }^{1}$ Principles of Radiation Therapy; available online at NCCN.org [NSCL-B]).

\section{Assessment of Treatment Response After Neoadjuvant Therapy}

Treatment response or downstaging of all tumors in the mediastinal lymph nodes is often predictive of a favorable outcome after surgical combined modality treatment. ${ }^{54-56}$ Conversely, the detection of disease progression after neoadjuvant therapy may avoid the cost and morbidity of local surgery for patients not likely to benefit.

Imaging plays an important role in assessing treatment response and restaging of NSCLC. Chest CT is usually performed before surgical resection, but the accuracy of CT scan in the evaluation of mediastinal lymph nodes is limited. ${ }^{57}$ FDG-PET seems to be an accurate noninvasive method to predict long-term outcome, and FDG-PET/CT is the best noninvasive modality for treatment response assessment, including evaluation of disease progression to new sites.

An early (1-2 months) posttreatment FDG-PET/CT scan is a prognostic factor for survival 
Martins et al.

and is more predictive than CT response, stage, or pretreatment performance status in patients treated with definitive chemoradiation. ${ }^{58}$ FDG uptake early during the course of chemotherapy was found to be predictive of progression-free and overall survival, ${ }^{59}$ and the metabolic response in FDG activity during the course of CRT (at 45 Gy) was significantly correlated with posttreatment response. ${ }^{60}$ In patients treated with neoadjuvant chemotherapy or chemoradiation, several series have reported that reduction FDG activity correlates with pathologic complete response and favorable prognosis. ${ }^{61,62}$ For example, a negative scan or a reduction in the standard uptake value (SUV) of more than $80 \%$ was the best predictive factor for a favorable outcome of further treatment, whereas progressive disease according to PET (new tumor manifestations or increasing SUV) was significantly correlated with an unfavorable outcome $(P=.005) .{ }^{63}$ However FDG-PET is less accurate in predicting residual disease in the mediastinum. A recent pooled analysis ${ }^{64}$ of 497 patients treated in 9 prospective neoadjuvant CRT studies reported that the ranges of sensitivity, specificity, positive predictive value, and negative predictive value for primary tumor response assessment were $80 \%$ to $100 \%, 0 \%$ to $100 \%, 42.9 \%$ to $100 \%$, and $66.7 \%$ to $100 \%$, respectively. Pooling data for N2 restaging after neoadjuvant response, the overall sensitivity was $63.8 \%$ (95\% CI, $53.3 \%-73.7 \%$ ) and overall specificity was $85.3 \%$ (95\% CI, 80.4\%-89.4\%).

Although FDG-PET is part of the postneoadjuvant evaluation and compared favorably with cross-sectional imaging in restaging after neoadjuvant therapy in the reviewed publications, the pooled results do not support the use of this noninvasive diagnostic approach as the only reassessment tool for mediastinal lymph node evaluation in routine clinical use. Pathologic confirmation is recommended whenever possible. Repeating a mediastinoscopy is technically challenging because of mediastinal fibrosis, which may be worsened by the administration of neoadjuvant CRT. To address this problem, using EBUS (+/- EUS) in the initial mediastinal evaluation and reserving the mediastinoscopy for after neoadjuvant therapy may be the preferred strategy.

\section{Results of NCCN Member Institution Surveys on Management of Stage IIIA NSCLC}

In an attempt to clarify the practice patterns of the NCCN Member Institutions, in 2010 the NCCN NSCLC Panel polled its members regarding diagnostic methods and treatment considerations in stage IIIA N2 disease. There were 25 responses from 21 member institutions (Table 5). When the member institution had 2 delegates, each counted as 0.5 of the vote.

For initial evaluation of the mediastinum before surgical resection, $80 \%$ of NCCN Member Institutions indicated they use EBUS (+/- EUS) and 20\% were using only mediastinoscopy. The institutions were split regarding the pathologic evaluation of mediastinal lymph nodes after neoadjuvant therapy to make a final decision regarding surgery, with $40.5 \%$ pathologically staging the mediastinum after neoadjuvant therapy versus $59.5 \%$ that did not. Regarding the choice of neoadjuvant therapy, the institutions were evenly split, with 50\% using neoadjuvant chemotherapy most of the time and 50\% using neoadjuvant CRT most of the time.

Most NCCN Member Institutions consider surgery in patients with a single lymph node station smaller than $3 \mathrm{~cm}(90.5 \%)$. However, $47.6 \%$ of institutions consider surgery in patients with more than one lymph node station involved (as long as no lymph node is larger than $3 \mathrm{~cm}$ ), and even fewer institutions $(16.7 \%)$ would consider surgery in patients with more than one lymph node station involved, even if the lymph node is larger than $3 \mathrm{~cm}$. Finally, the $21 \mathrm{NCCN}$ Member Institutions were almost evenly split regarding the treatment of patients who require pneumonectomy; $54.8 \%$ of the institutions would consider neoadjuvant therapy followed by surgery when a patient is likely, based on initial evaluation, to require a pneumonectomy. This approach seems to be in contrast with the results of INT0139, which showed that patients treated with pneumonectomy had a worse outcome. However, several recent retrospective reviews indicate that pneumonectomy can be safely performed after neoadjuvant therapy, particularly in patients treated with chemotherapy only. ${ }^{65-67}$

The differences in approach between NCCN Member Institutions are most evident in the issues of the choice between neoadjuvant chemotherapy or 
Management of Stage IIIA (N2) NSCLC

\section{Table 5 NCCN Member Poll: Collated Responses by Institution ${ }^{2}$}

\begin{tabular}{|c|c|c|}
\hline Poll Question & Yes (\%) & No (\%) \\
\hline $\begin{array}{l}\text { Does your institution use EBUS (+/-EUS) in the initial evaluation of the mediastinum and } \\
\text { perform the first mediastinoscopy before surgical resection? }\end{array}$ & $16(80)$ & $4(20)$ \\
\hline $\begin{array}{l}\text { Does your institution use pathologic evaluation of mediastinal LNs, after neoadjuvant } \\
\text { therapy, to make a final decision regarding surgery? }\end{array}$ & $8.5(40.5)$ & $12.5(59.5)$ \\
\hline $\begin{array}{l}\text { When using neoadjuvant therapy, does your institution use neoadjuvant chemotherapy } \\
\text { most of the time? }\end{array}$ & $10.5(50)$ & $10.5(50)$ \\
\hline $\begin{array}{l}\text { When using neoadjuvant therapy, does your institution use neoadjuvant } \\
\text { chemoradiotherapy most of the time? }\end{array}$ & $10.5(50)$ & $10.5(50)$ \\
\hline Does your institution consider surgery in patients with a single $L N$ station $<3 \mathrm{~cm}$ ? & $19(90.5)$ & $2(9.5)$ \\
\hline $\begin{array}{l}\text { Does your institution consider surgery in patients with more than } 1 \mathrm{LN} \text { station as long as no } \\
\mathrm{LN} \text { is }>3 \mathrm{~cm} \text { ? }\end{array}$ & $10(47.6)$ & $11(52.4)$ \\
\hline $\begin{array}{l}\text { Does your institution consider surgery in patients with more than one LN station involved, } \\
\text { even if the LN is }>3 \mathrm{~cm} \text { ? }\end{array}$ & $3.5(16.7)$ & $17.5(83.3)$ \\
\hline $\begin{array}{l}\text { Does your institution consider neoadjuvant therapy followed by surgery when a patient is } \\
\text { likely, based on initial evaluation, to require a pneumonectomy? }\end{array}$ & $11.5(54.8)$ & $9.5(45.2)$ \\
\hline
\end{tabular}

Abbreviations: EBUS, endobronchial ultrasound; EUS, endoscopic ultrasound; LN, lymph node. aTwenty-five responses were received from 21 NCCN Member Institutions. Two responses each were received from City of Hope Comprehensive Cancer Center, Roswell Park Cancer Institute, and Fox Chase Cancer Center. Separate responses were received from Dana-Farber Cancer Institute and Massachusetts General Hospital Cancer Center; the same vote counted as 1, a split vote counted as 0.5 in each column.

CRT, the role of surgery in patients with more than one lymph node station involved, and in those requiring a pneumonectomy. These differences can only be resolved with randomized prospective trials. However, considering the difficulties in accrual to trials of multimodality therapy in stage IIIA, these issues are unlikely to be resolved in the near future.

\section{Conclusions}

The management of patients with stage IIIA with N2 nodal involvement represents the most controversial issue in the therapy of NSCLC. Although randomized phase III trials failed to show a survival benefit from surgery after chemotherapy or CRT, they do not account for the heterogeneity of N2 disease and are compromised by imprecise staging, slow patient accru$\mathrm{al}$, and poor surgical outcomes, perhaps because of surgery performed by surgeons without thoracic surgery specialization. Decisions regarding the role of surgery, as a part of the treatment plan, should be made before the initiation of therapy, and patients most likely to benefit from its inclusion are those with single station, non-bulky $(<3 \mathrm{~cm})$ nodal involvement. There is no consensus regarding the best neoadjuvant approach, and chemotherapy or CRT are both reasonable choices. Radiotherapy techniques have evolved and it is possible to increase doses while minimizing additional toxicity, potentially leading to a therapeutic improvement. The presence of a dedicated thoracic oncology multidisciplinary team is key to making optimum and individualized therapeutic decisions for these patients, and offers the best opportunity for long-term survival.

\section{Acknowledgments}

The authors would like to thank Lisa White (FHCRC/SCCA/UW) for assistance during the manuscript preparation process.

\section{References}

1. Ettinger DS, Akerly W, Bepler G, et al. NCCN Clinical Practice Guidelines in Oncology: Non-Small Cell Lung Cancer. Version 2, 2012. Available at: NCCN.org. Accessed March 13, 2012.

2. Goldstraw P, Crowley J, Chansky K, et al. The IASLC lung cancer staging project: proposals for the revision of the TNM stage groupings in the forthcoming (seventh) edition of the TNM classification of malignant tumors. J Thorac Oncol 2007;2:706-714.

3. Albain KS, Swann RS, Rusch VW, et al. Radiotherapy plus chemotherapy with or without surgical resection for stage III nonsmall-cell lung cancer: a phase III randomized controlled trial. Lancet 2009;374:379-386

4. Shah $\mathrm{AA}$, Berry MF, Tzao C, et al. Induction chemoradiotherapy is not superior to induction chemotherapy alone in patients with stage IIIA(N2) non-small cell lung cancer: a systematic review and meta- 
Martins et al.

analysis [abstract]. J Thorac Oncol 2011;6(Supp 2):S1578-1579. Abstract 4.290.

5. Andre F, Grunenwald D, Pignon JP, et al. Survival of patients with resected $\mathrm{N} 2$ non-small-cell lung cancer: evidence for a subclassification and implications. J Clin Oncol 2000;18:2981-2989.

6. Cerfolio RJ, Bryant AS. Survival of patients with unsuspected N2 (stage IIIA) nonsmall-cell lung cancer. Ann Thorac Surg 2008;86:362-367.

7. Hochstenbag MMH, Twijnstra A, Hofman P, et al. MR-imaging of the brain of neurologic asymptomatic patients with large cell or adenocarcinoma of the lung. Does it influence prognosis and treatement? Lung Cancer 2003;42:189-193.

8. Hwangbo B, Kim SK, Lee HS, et al. Application of endobronchial ultrasound-guided transbronchial needle aspiration following integrated PET/CT in mediastinal staging of potentially operable nonsmall cell lung cancer. Chest 2009;135:1280-1287.

9. Darling GE, Maziak DE, Inculet RI, et al. Positron emission tomography-computed tomography compared with invasive mediastinal staging in non-small cell lung cancer: results of mediastinal staging in the early lung positron emission tomography trial. J Thorac Oncol 2011;6:1367-1372.

10. Farjah F, Flum DR, Varguese TK Jr, et al. Surgeon specialty and longterm survival after pulmonary resection for lung cancer. Ann Thorac Surg 2008;87:995-1004.

11. Burkes RL, Ginsberg RJ, Shepherd FA, et al. Induction chemotherapy with mitomycin, vindesine, and cisplatin for stage III unresectable non-small-cell lung cancer: results of the Toronto phase II trial. J Clin Oncol 1992;10:580-586.

12. Martini N, Kris MG, Flehinger BJ, et al. Preoperative chemotherapy for stage IIIa (N2) lung cancer: the Sloan-Kettering experience with 136 patients. Ann Thorac Surg 1993;55:1365-1373; discussion 1373 1374.

13. Elias AD, Skarin AT, Leong T, et al. Neoadjuvant therapy for surgically staged IIIA N2 non-small cell lung cancer (NSCLC). Lung Cancer 1997;17:147-161.

14. Pujol JL, Rossi JF, Le Chevalier T, et al. Pilot study of neoadjuvant ifosfamide, cisplatin, and etoposide in locally advanced non-small cell lung cancer. Eur J Cancer 1990;26:798-801.

15. Darwish S, Minotti V, Crino L, et al. Neoadjuvant cisplatin and etoposide for stage IIIA (clinical N2) non-small cell lung cancer. Am J Clin Oncol 1994;17:64-67.

16. Kris MG, Pisters KM, Ginsberg RJ, et al. Effectiveness and toxicity of preoperative therapy in stage IIIA non-small cell lung cancer including the Memorial Sloan-Kettering experience with induction MVP in patients with bulky mediastinal lymph node metastases (clinical N2). Lung Cancer 1995;12(Suppl 1):S47-57.

17. Betticher DC, Hsu Schmitz SF, Totsch M, et al. Mediastinal lymph node clearance after docetaxel-cisplatin neoadjuvant chemotherapy is prognostic of survival in patients with stage IIIA pN2 non-small-cell lung cancer: a multicenter phase II trial. J Clin Oncol 2003;21:17521759.

18. Martini N, Kris MG, Gralla RJ, et al. The effects of preoperative chemotherapy on the resectability of non-small cell lung carcinoma with mediastinal lymph node metastases (N2 M0). Ann Thorac Surg 1988;45:370-379.

19. Sugarbaker DJ, Herndon J, Kohman LJ, et al. Results of cancer and leukemia group B protocol 8935. A multiinstitutional phase II trimodality trial for stage IIIA (N2) non-small-cell lung cancer. Cancer and Leukemia Group B Thoracic Surgery Group. J Thorac Cardiovasc Surg 1995;109:473-483; discussion 483-485.

20. Burkes RL, Shepherd FA, Blackstein ME, et al. Induction chemotherapy with mitomycin, vindesine, and cisplatin for stage IIIA
(T1-3, N2) unresectable non-small-cell lung cancer: final results of the Toronto phase II trial. Lung Cancer 2005;47:103-109.

21. Garrido P, Gonzalez-Larriba JL, Insa A, et al. Long-term survival associated with complete resection after induction chemotherapy in stage IIIA (N2) and IIIB (T4N0-1) non small-cell lung cancer patients: the Spanish Lung Cancer Group Trial 9901. J Clin Oncol 2007;25:4736-4742.

22. Weiden PL, Piantadosi S. Preoperative chemotherapy (cisplatin and fluorouracil) and radiation therapy in stage III non-small cell lung cancer. A phase 2 study of the LCSG. Chest 1994;106(6 Suppl):344S$347 \mathrm{~S}$.

23. Taylor SG IV, Trybula M, Bonomi PD, et al. Simultaneous cisplatin fluorouracil infusion and radiation followed by surgical resection in regionally localized stage III, non-small cell lung cancer. Ann Thorac Surg 1987;43:87-91.

24. D'Angelillo RM, Trodella L, Ciresa M, et al. Multimodality treatment of stage III non-small cell lung cancer: analysis of a phase II trial using preoperative cisplatin and gemcitabine with concurrent radiotherapy. J Thorac Oncol 2009;4:1517-1523.

25. Palazzi M, Cataldo I, Gramaglia A, et al. Preoperative concomitant cisplatin/VP16 and radiotherapy in stage III non-small cell lung cancer. Int J Radiat Oncol Biol Phys 1993;27:621-625.

26. Choi NC, Carey RW, Daly W, et al. Potential impact on survival of improved tumor downstaging and resection rate by preoperative twicedaily radiation and concurrent chemotherapy in stage IIIA non-smallcell lung cancer. J Clin Oncol 1997;15:712-722.

27. Favaretto A, Paccagnella A, Tomio L, et al. Pre-operative chemoradiotherapy in non-small cell lung cancer stage III patients. Feasibility, toxicity and long-term results of a phase II study. Eur J Cancer 1996;32A:2064-2069.

28. Eberhardt W, Wilke H, Stamatis G, et al. Preoperative chemotherapy followed by concurrent chemoradiation therapy based on hyperfractionated accelerated radiotherapy and definitive surgery in locally advanced non-small-cell lung cancer: mature results of a phase II trial. J Clin Oncol 1998;16:622-634.

29. Strauss GM, Herndon JE, Sherman DD, et al. Neoadjuvant chemotherapy and radiotherapy followed by surgery in stage IIIA nonsmall-cell carcinoma of the lung: report of a Cancer and Leukemia Group B phase II study. J Clin Oncol 1992;10:1237-1244.

30. Eagan RT, Ruud C, Lee RE, et al. Pilot study of induction therapy with cyclophosphamide, doxorubicin, and cisplatin (CAP) and chest irradiation prior to thoracotomy in initially inoperable stage III MO non-small cell lung cancer. Cancer Treat Rep 1987;71:895-900.

31. Faber LP, Kittle CF, Warren WH, et al. Preoperative chemotherapy and irradiation for stage III non-small cell lung cancer. Ann Thorac Surg 1989;47:669-675; discussion 676-677.

32. Recine D, Rowland $\mathrm{K}$, Reddy $\mathrm{S}$, et al. Combined modality therapy for locally advanced non-small cell lung carcinoma. Cancer 1990;66:2270-2278.

33. Thomas M, Rübe C, Hoffnecht $\mathrm{P}$, et al. Effect of preoperative chemoradiation in addition to preoperative chemotherapy: a randomised trial in stage III non-small cell lung cancer. Lancet Oncol 2008;9:636-648.

34. Preoperative chemotherapy versus concurrent chemoradiotherapy in N2 positive IIIa non small cell lung cancer (NSCLC). ClinicalTrials. gov identifier: NCT00452803. Available at: www.clinicaltrials.gov/ NCT00452803. Accessed November 21, 2011.

35. Chemotherapy with or without radiation therapy before surgery in treating patients with stage IIIA non-small cell lung cancer. Clinical Trials.gov identifier: NCT00030771. Available at: www.clinicaltrials. gov/NCT00030771. Accessed November 21, 2011. 
36. van Meerbeeck JP, Kramer GW, Van Schil PE, et al. Randomized controlled trial of resection versus radiotherapy after induction chemotherapy in stage IIIA-N2 non-small-cell lung cancer. J Natl Cancer Inst 2007;99:442-450.

37. Mac Manus MP, Hicks RJ, Matthew JP, et al., High rate of detection of unsuspected distant metastases by PET in apparent stage III nonsmall-cell lung cancer: implications for radical radiation therapy. Intl J Radiat Oncol Biol Phys 2001;50:287-293.

38. Fischer B, Lassen U, Mortensen J, et al. Preoperative staging of lung cancer with combined PET-CT. N Engl J Med 2009;361:32-39.

39. Farray D, Mirkovic N, Albain KS. Multimodality therapy for stage III non-small cell lung cancer. J Clin Oncol 2005;23:7763.

40. Aupérin A, Le Péchoux C, Rolland E, et al. Meta-analysis of concomitant versus sequential radiochemotherapy in locally advanced non-small-cell lung cancer. J Clin Oncol 2010;28:2181-2190.

41. Cerfolio R, A Bryant, B Ojha. Restaging patients with N2 (stage IIIA) nonsmall cell lung cancer after neoadjuvant chemoradiotherapy: a prospective study. J Thorac Cardiovasc Surg 2006;131:1229-1235.

42. Bauwens $\mathrm{O}$, Dusart M, Pierard P, et al. Endobronchial ultrasound and value of PET for prediction of pathological results of mediastinal hot spots in lung cancer patients. Lung Cancer 2008;61:356-361.

43. Plat G, Pierard P, Haller A, et al. Endobronchial ultrasound and positron emission tomography positive mediastinal lymph nodes. Eur Respir J 2006;27:276-281.

44. Krupitskaya Y, Loo BW Jr. Post-operative radiation therapy (PORT) in completely resected non-small-cell lung cancer. Curr Treat Opt Oncol 2008;9:343-356.

45. Watanabe Y, Shimizu J, Tsubota M, et al. Mediastinal spread of metastatic lymph nodes in bronchogenic carcinoma. Mediastinal nodal metastases in lung cancer. Chest 1990;97:1059-1065.

46. Kotoulas CS, Foroulis CN, Kostikas K, et al. Involvement of lymphatic metastatic spread in non-small cell lung cancer accordingly to the primary cancer location. Lung Cancer 2004;44:183-191.

47. Cerfolio RJ, Bryant AS. Distribution and likelihood of lymph node metastasis based on the lobar location of nonsmall-cell lung cancer. Ann Thorac Surg 2006;81:1969-1973.

48. Kelsey CR, Light KL, Marks LB. Patterns of failure after resection of non-small-cell lung cancer: implications for postoperative radiation therapy volumes. Int J Radiat Oncol Biol Phys 2006;65:1097-1105.

49. Fowler WC, Langer CJ, Curran WJ, et al. Postoperative complications after combined neoadjuvant treatment of lung cancer. Ann Thorac Surg 1993;55:986-989.

50. Sonett JR, Suntharalingam M, Edelman MJ, et al. Pulmonary resection after curative intent radiotherapy ( $>59 \mathrm{~Gy}$ ) and concurrent chemotherapy in non-small-cell lung cancer. Ann Thorac Surg 2004;78:1200-1205.

51. Cerfolio RJ, Bryant AS, Jones VL, et al. Pulmonary resection after concurrent chemotherapy and high dose (60Gy) radiation for nonsmall cell lung cancer is safe and may provide increased survival. Eur J Cardiothorac Surg 2009;35:718-723.

52. Edelman MJ, Paulus R, Suntharalingam M, et al. RTOG 0229: a phase II trial of concurrent chemotherapy and full dose radiotherapy followed by surgical resection and consolidative therapy for locally advanced non-small cell carcinoma of the lung [abstract]. J Thorac Oncol 2011;6:S375-376. Abstract O23.02.

53. Spoelstra FOB, Senan S, Le Péchoux C, et al. Variations in target volume definition for postoperative radiotherapy in stage III nonsmall-cell lung cancer: analysis of an international contouring study. Int J Radiat Oncol Biol Phys 2010;76:1106-1113.

54. Betticher DC, Hsu Schmitz SF, Tötsch M, et al. Prognostic factors affecting long-term outcomes in patients with resected stage IIIA pN2 non-small-cell lung cancer: 5-year follow-up of a phase II study. Br J Cancer 2006;94:1099-1106.

55. Stefani A, Alifano M, Bobbio A, et al. Which patients should be operated on after induction chemotherapy for N2 non-small cell lung cancer? Analysis of a 7-year experience in 175 patients. J Thorac Cardiovasc Surg 2010;140:356-363.

56. Kim AW, Liptay MJ, Bonomi $\mathrm{P}$, et al. Neoadjuvant chemoradiation for clinically advanced non-small cell lung cancer: an analysis of 233 patients. Ann Thorac Surg 2011;92:233-241; discussion 241-243.

57. Lee HY, Lee HJ, Kim YT, et al. Value of combined interpretation of computed tomography response and positron emission tomography response for prediction of prognosis after neoadjuvant chemotherapy in non-small cell lung cancer. J Thorac Oncol 2010;5:497-503.

58. Mac Manus M, Hicks RJ. The use of positron emission tomography (PET) in the staging/evaluation, treatment, and follow-up of patients with lung cancer: a critical review. Int J Radiat Oncol Biol Phys 2008;72:1298-1306.

59. Dimitrakopoulou-Strauss A, Hoffman M, Bergner R, et al. Prediction of short-term survival in patients with advanced nonsmall cell lung cancer following chemotherapy based on 2-deoxy-2-[F-18]fluoro-Dglucose-positron emission tomography: a feasibility study. Mol Imaging Biol 2007;9:308-317.

60. Kong FM, Frey KA, Quint LE, et al. A pilot study of [18F] fluorodeoxyglucose positron emission tomography scans during and after radiation-based therapy in patients with non small-cell lung cancer. J Clin Oncol 2007;25:3116-3123.

61. Cerfolio RJ, Bryant AS, Winokur TS, et al. Repeat FDG-PET after neoadjuvant therapy is a predictor of pathologic response in patients with non-small cell lung cancer. Ann Thorac Surg 2004;78:19031909; discussion 1909.

62. Ohtsuka T, Nomori H, Ebihara A, et al. FDG-PET imaging for lymph node staging and pathologic tumor response after neoadjuvant treatment of non-small cell lung cancer. Ann Thorac Cardiovasc Surg 2006;12:89-94.

63. Eschmann SM, Friedel G, Paulsen F, et al. 18F-FDG PET for assessment of therapy response and preoperative re-evaluation after neoadjuvant radio-chemotherapy in stage III non-small cell lung cancer. Eur J Nucl Med Mol Imaging 2007;34:463-471.

64. Rebollo-Aguirre AC, Ramos-Font C, Villegas Portero R, et al. Is FDGPET suitable for evaluating neoadjuvant therapy in non-small cell lung cancer? Evidence with systematic review of the literature. J Surg Oncol 2010;101:486-494.

65. Gaissert HA, Keum DY, Wright CD, et al. Operative risk of pneumonectomy-Influence of preoperative induction therapy. J Thorac Cardiovasc Surg 2009;138:289-294.

66. Mansour Z, Kochetkova EA, Ducrocq E, et al. Induction chemotherapy does not increase the operative risk of pneumonectomy. Eur J Cardiothorac Surg 2007;31:181-185.

67. Weder W, Collaud S, Eberhardt WEE, et al. Pneumectomy is a valuable treatment option after neoadjuvant therapy for stage III non-smallcell lung cancer. J Thorac Cardiovasc Surg 2010;139:1424-1430. 